\title{
Transport Properties of Short Alkyl Chain Length Dicationic Ionic Liquids - The Effects of Alkyl Chain Length and Temperature
}

\author{
Majid Moosavi $^{a, *}$, Fatemeh Khashei ${ }^{a}$, Ali Sharifi ${ }^{b}$, Mojtaba Mirzaei $^{\text {b }}$ \\ ${ }^{\text {a }}$ Dept. of Chemistry, University of Isfahan, Isfahan 81746-73441, Iran \\ ${ }^{\mathrm{b}}$ Chemistry and Chemical Engineering Research Center of Iran, P.O. Box 14335-186, Tehran, Iran \\ *Corresponding author. E-mail: m.mousavi@sci.ui.ac.ir \\ Tel.: +98-313-7934942; Fax: +98-313-668-9732
}


$\underline{{ }^{1} \mathrm{H}-\mathrm{NMR} \text { (at T }=60^{\circ} \mathrm{C} \text { ), elemental analysis and water contents of the studied dicationic ILs: }}$

a) 1,3-bis(3-methylimidazolium-1-yl)propane bis(trifluoromethylsulfonyl)imide $\left[\mathrm{C}_{3}(\mathrm{mim})_{2}\right]\left[\mathrm{NTf}_{2}\right]_{2}$ :

${ }^{1} \mathrm{H}$ NMR (400 MHz): $\delta$ (ppm) 1.91(m, 2H), 3.24(s, 6H), 3.66(m, 4H), 6.75(s, 2H), 6.82(s, 2H), 7.90(s, 2H). ${ }^{13} \mathrm{C}$ NMR (100 MHz): $\delta$ (ppm) 26.14, 35.84, 48.24, 122.32, 123.81, 136.71. ${ }^{19} \mathrm{~F}$ NMR (376 MHz): $\delta(\mathrm{ppm})-80.45$. Elemental analysis: calculated (\%) for $\mathrm{C}_{15} \mathrm{H}_{18} \mathrm{~F}_{12} \mathrm{~N}_{6} \mathrm{O}_{8} \mathrm{~S}_{4}$ : C 23.50, H 2.37, N 10.96, $\mathrm{S}$ 16.73; found: $\mathrm{C} 23.64, \mathrm{H}$ 2.25, N 10.96, S 16.89. Water content: 184 ppm.
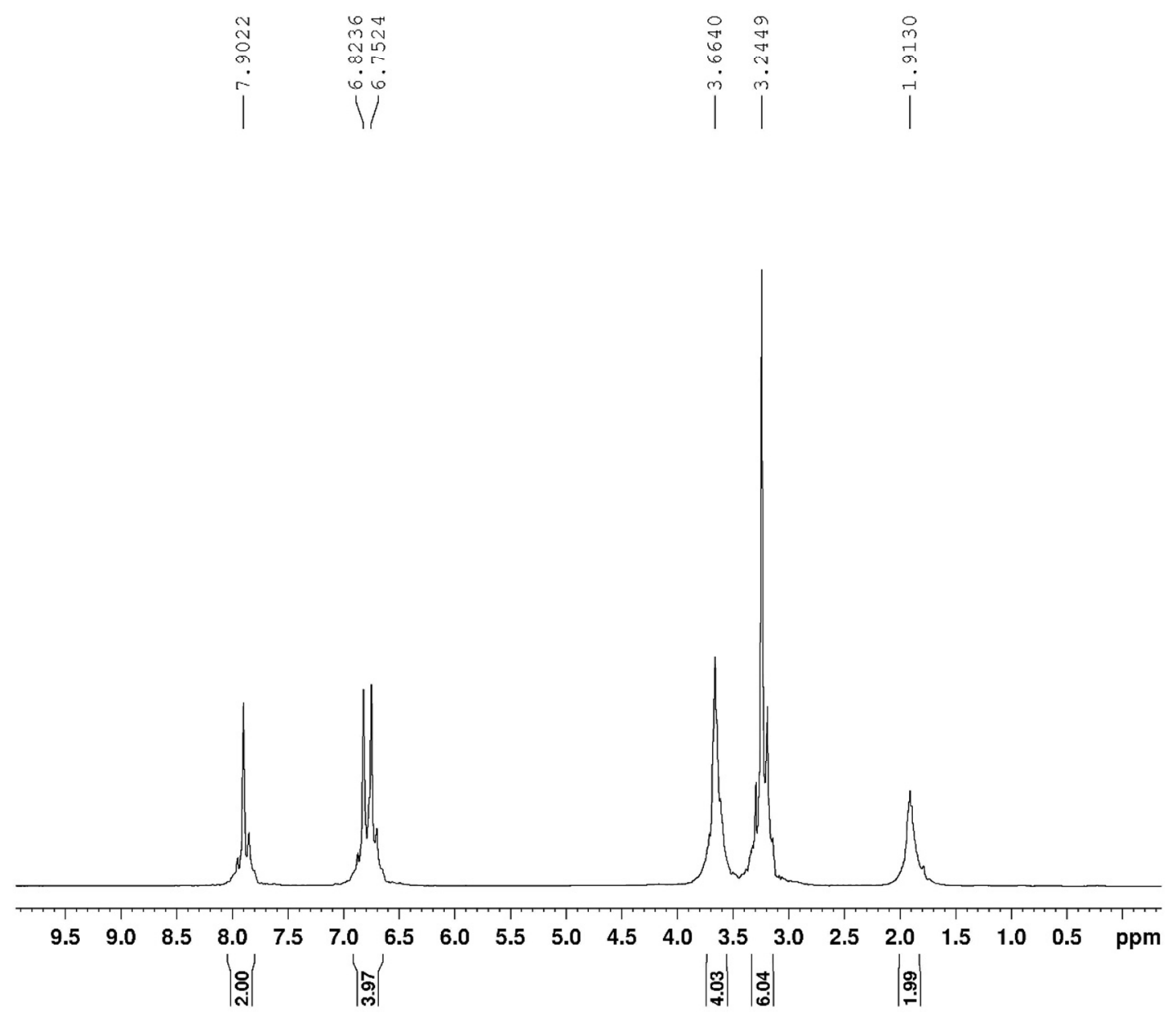


\section{b) 1,4-bis(3-methylimidazolium-1-yl)butane bis(trifluoromethylsulfonyl)imide}

$\left[\mathrm{C}_{4}(\mathrm{mim})_{2}\right]\left[\mathrm{NTf}_{2}\right]_{2}$ :

${ }^{1} \mathrm{H}$ NMR (400 MHz): $\delta$ (ppm) 1.32(m, 4H), 3.24(s, 6H), 3.59(m, 4H), 6.71(s, 2H), 6.78(s, 2H), 7.85(s, 2H). ${ }^{13} \mathrm{C}$ NMR (100 MHz): $\delta$ (ppm) 29.50, 35.84, 46.00, 122.30, 123.86, 136.93. ${ }^{19} \mathrm{~F}$ NMR (376 MHz): $\delta(\mathrm{ppm})-80.45$. Elemental analysis: calculated (\%) for $\mathrm{C}_{16} \mathrm{H}_{20} \mathrm{~F}_{12} \mathrm{~N}_{6} \mathrm{O}_{8} \mathrm{~S}_{4}$ : C 24.62, H 2.58, N 10.77, $\mathrm{S} 16.43$; found: $\mathrm{C} 24.75, \mathrm{H}$ 2.49, N 10.74, S 16.37. Water content: 182 ppm.
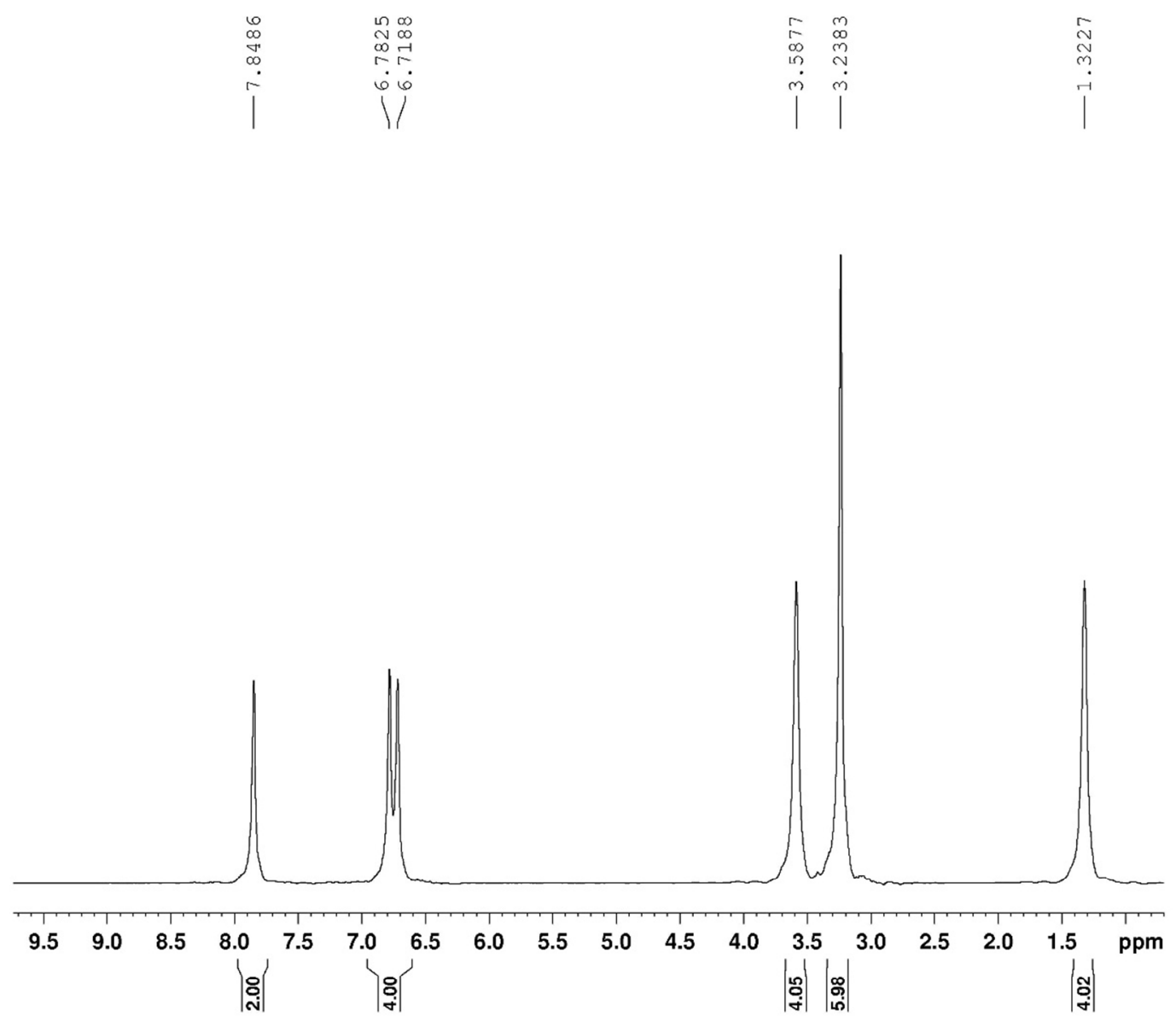


\section{c) 1,5-bis(3-methylimidazolium-1-yl)pentane bis(trifluoromethylsulfonyl)imide}

\section{$\left[\mathrm{C}_{5}(\mathrm{mim})_{2}\right]\left[\mathrm{NTf}_{2}\right]_{2}$ :}

${ }^{1} \mathrm{H}$ NMR (400 MHz): $\delta(\mathrm{ppm})$ 0.75(m, 2H), 1.297(m, 4H), 3.23(s, 6H), 3.54(m, 4H), 6.71(s, 2H), 6.78(s, 2H), 7.85 (s, 2H). ${ }^{13} \mathrm{C} \mathrm{NMR} \mathrm{(100} \mathrm{MHz):} \delta(\mathrm{ppm}) 21.68,28.20$, 35.10, 48.75, 121.72, 123.15, 135.46. ${ }^{19} \mathrm{~F}$ NMR (376 MHz): $\delta(\mathrm{ppm})-80.43$. Elemental analysis: calculated (\%) for $\mathrm{C}_{17} \mathrm{H}_{22} \mathrm{~F}_{12} \mathrm{~N}_{6} \mathrm{O}_{8} \mathrm{~S}_{4}$ : C 25.70, H 2.79, N 10.58, S 16.14; found: C 25.85, H 2.85, N 11.00, S 16.40. Water content: 176 ppm.

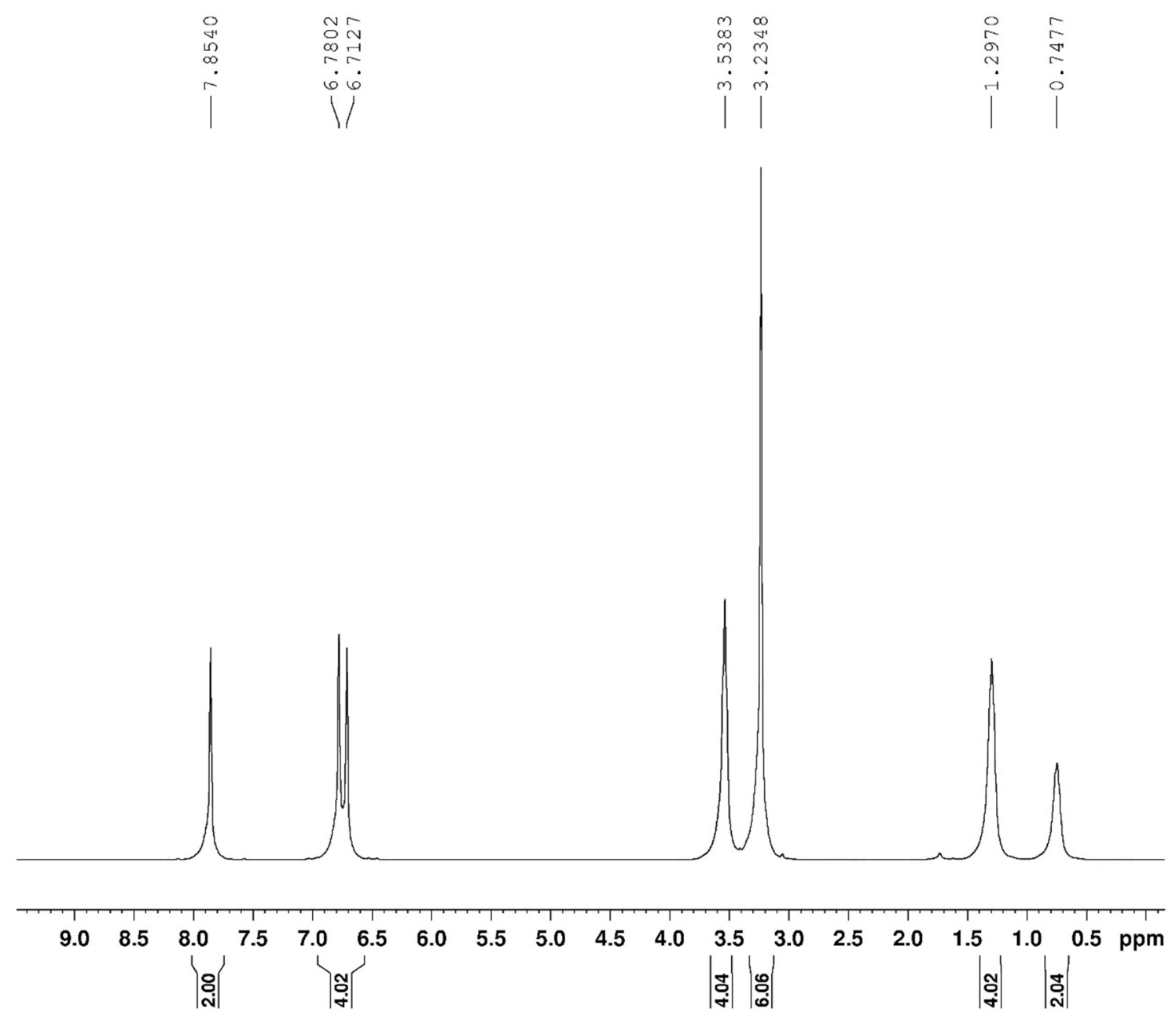




\section{Figures:}
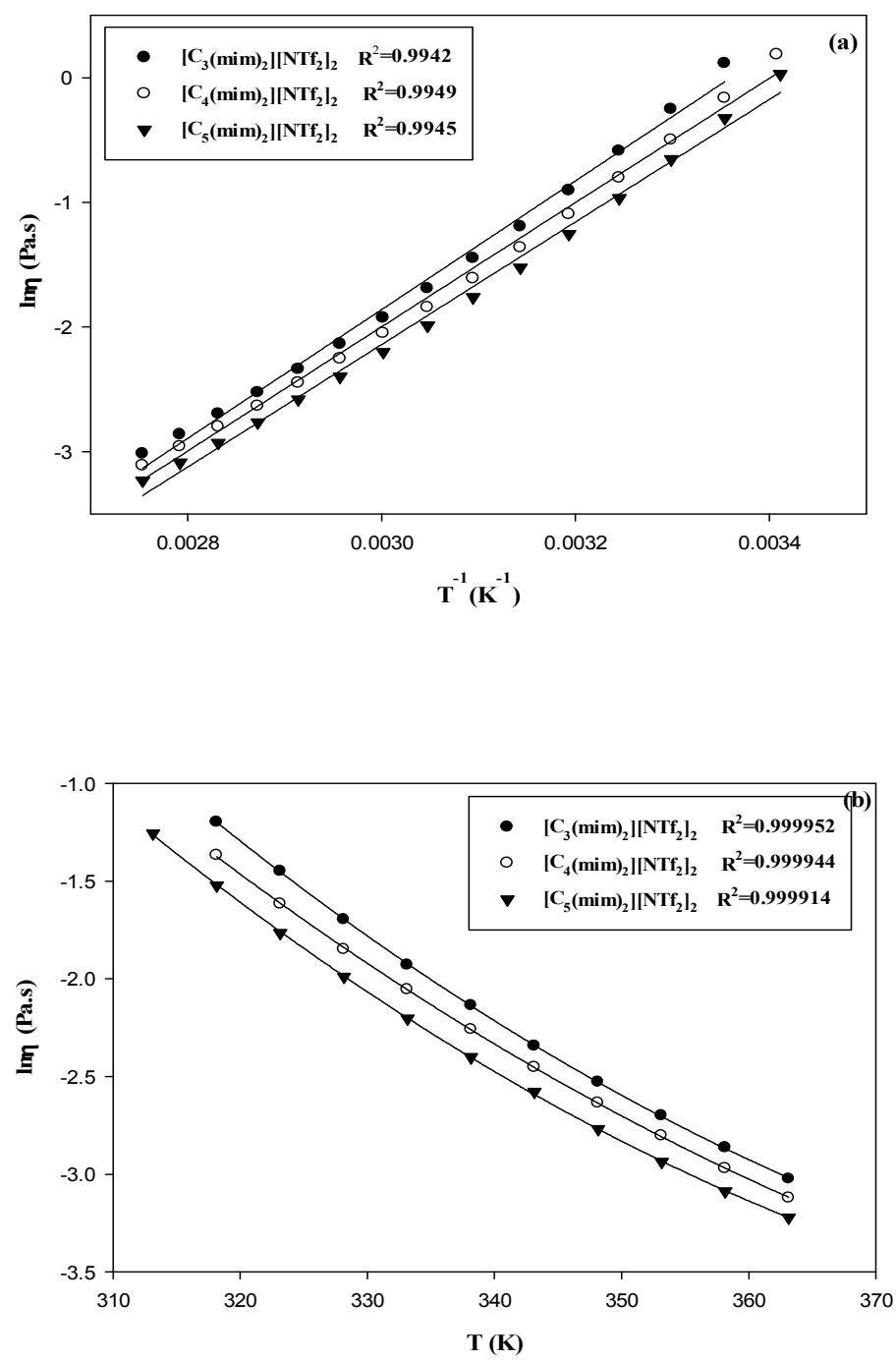

Figure S1. (a) $\ln \eta$ versus $1 / T$ profiles and (b) $\ln \eta$ versus $T$ profiles at shear rate $=14 \mathrm{~s}^{-1}$ for the studied DILs. 

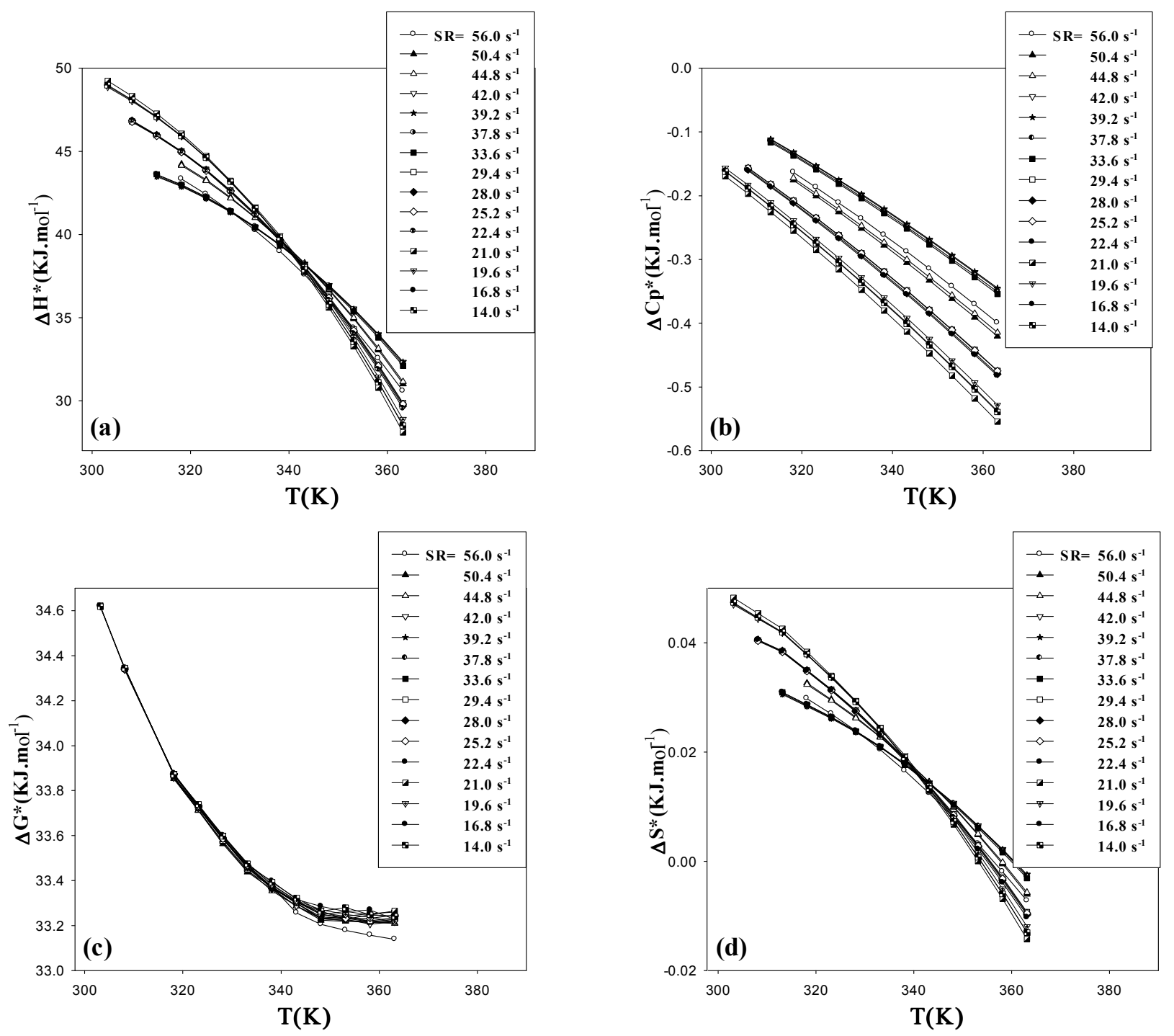

Figure S2. Activation parameters at different temperatures and shear rates for $\left[\mathrm{C}_{3}(\operatorname{mim})_{2}\right]\left[\mathrm{NTf}_{2}\right]_{2}$, (a) $\Delta G^{*}$ profiles (b) $\Delta H^{*}$ profiles, (c) $\Delta S^{*}$ profiles, and (d) $\Delta C_{p}^{*}$ profiles. 

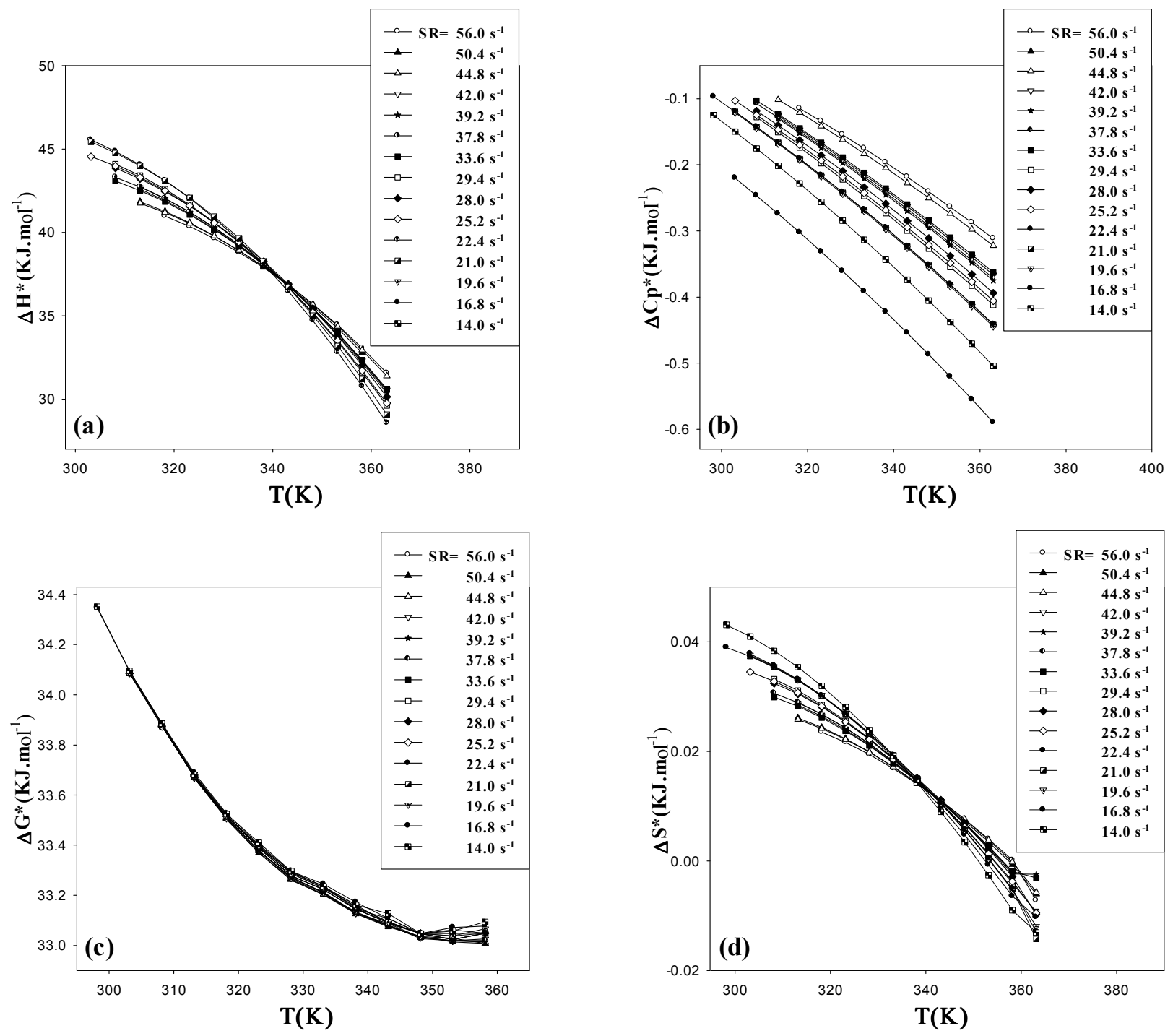

Figure S3. The same as Fig. S2 for $\left[\mathrm{C}_{4}(\mathrm{mim})_{2}\right]\left[\mathrm{NTf}_{2}\right]_{2}$. 

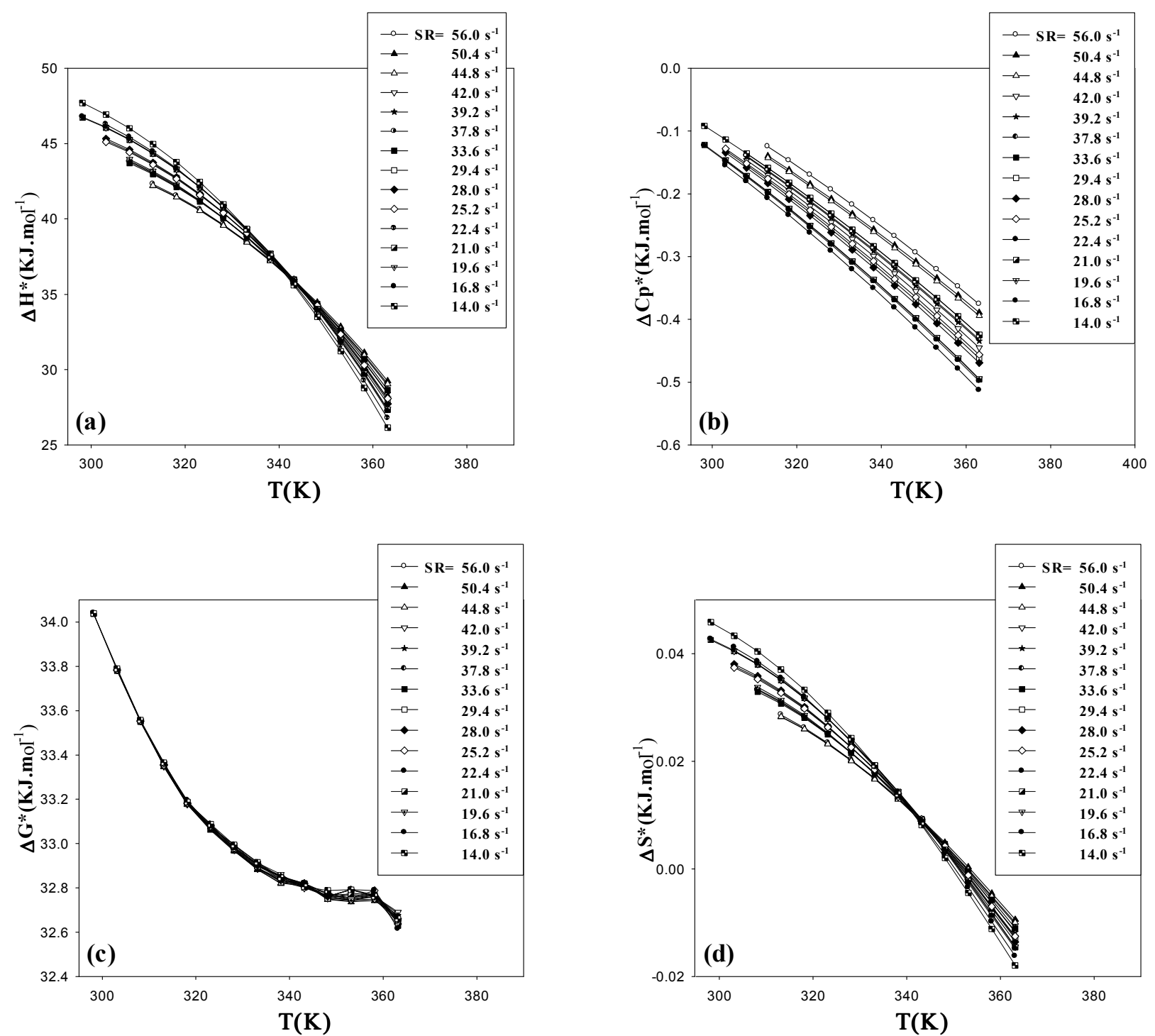

Figure S4. The same as Fig. S2 for $\left[\mathrm{C}_{5}(\mathrm{mim})_{2}\right]\left[\mathrm{NTf}_{2}\right]_{2}$. 
Tables:

Table S1. Y-Intercept and Slope Values of Regression Lines Drawn through Plots of Shear Stress against Shear Rate for $\left[\mathrm{C}_{3}(\mathrm{mim})_{2}\right]\left[\mathrm{NTf}_{2}\right]_{2},\left[\mathrm{C}_{4}(\mathrm{mim})_{2}\right]\left[\mathrm{NTf}_{2}\right]_{2},\left[\mathrm{C}_{5}(\mathrm{mim})_{2}\right]\left[\mathrm{NTf}_{2}\right]_{2}$ DILs

\begin{tabular}{|c|c|c|c|c|c|c|}
\hline \multirow{2}{*}{$\mathrm{T} /{ }^{\circ} \mathrm{C}$} & \multicolumn{2}{|c|}{$\left[\mathrm{C}_{3}(\mathrm{mim})_{2}\right]\left[\mathrm{NTf}_{2}\right]_{2}$} & \multicolumn{2}{|c|}{$\left[\mathrm{C}_{4}(\mathrm{mim})_{2}\right]\left[\mathrm{NTf}_{2}\right]_{2}$} & \multicolumn{2}{|c|}{$\left[\mathrm{C}_{5}(\mathrm{mim})_{2}\right]\left[\mathrm{NTf}_{2}\right]_{2}$} \\
\hline & intercept & slope & intercept & slope & intercept & slope \\
\hline 25 & - & - & 1.4000 & 8.3929 & 0.1259 & 7.2278 \\
\hline 30 & -0.1237 & 7.7772 & 0.8122 & 6.0271 & 0.3431 & 5.1795 \\
\hline 35 & -0.5724 & 5.5832 & 0.5741 & 4.4506 & 0.0269 & 3.8069 \\
\hline 40 & 0.3456 & 4.0341 & 0.5795 & 3.3213 & 0.5128 & 2.8368 \\
\hline 45 & 0.7215 & 3.0029 & 0.4486 & 2.5416 & 0.1883 & 2.1730 \\
\hline 50 & 0.3297 & 2.3404 & 0.6573 & 1.9749 & 0.3175 & 1.7071 \\
\hline 55 & 0.4139 & 1.8261 & 0.4540 & 1.5681 & 0.3149 & 1.3608 \\
\hline 60 & 0.3011 & 1.4467 & 0.5098 & 1.2715 & 0.2619 & 1.0984 \\
\hline 65 & 0.1490 & 1.1744 & 0.3377 & 1.0378 & 0.2991 & 0.8994 \\
\hline 70 & 0.2972 & 0.9543 & 0.2333 & 0.8559 & -0.0112 & 0.7586 \\
\hline 75 & 0.2714 & 0.7899 & 0.1287 & 0.7138 & 0.1550 & 0.6251 \\
\hline 80 & 0.2072 & 0.6671 & 0.2329 & 0.5995 & 0.1973 & 0.5272 \\
\hline 85 & 0.2044 & 0.5648 & 0.2721 & 0.5086 & 0.0676 & 0.4537 \\
\hline 90 & 0.2011 & 0.4829 & 0.2271 & 0.4377 & -0.1286 & 0.4002 \\
\hline
\end{tabular}


Table S2. Experimental Data of Viscosity (mPa.s) of the Studied DILs*

\begin{tabular}{cccc}
\hline & \multicolumn{3}{c}{ DILs } \\
\cline { 2 - 4 } $\mathrm{T}(\mathrm{K})$ & {$\left[\mathrm{C}_{3}(\mathrm{mim})_{2}\right]\left[\mathrm{NTf}_{2}\right]_{2}$} & {$\left[\mathrm{C}_{4}(\mathrm{mim})_{2}\right]\left[\mathrm{NTf}_{2}\right]_{2}$} & {$\left[\mathrm{C}_{5}(\mathrm{mim})_{2}\right]\left[\mathrm{NTf}_{2}\right]_{2}$} \\
\hline 293.15 & --- & 1203.00 & 1029.00 \\
298.15 & 1122.00 & 848.97 & 723.42 \\
303.15 & 776.70 & 606.95 & 519.60 \\
308.15 & 555.95 & 447.55 & 380.81 \\
313.15 & 404.88 & 334.26 & 285.47 \\
318.15 & 302.89 & 255.78 & 218.01 \\
323.15 & 235.28 & 199.89 & 171.90 \\
328.15 & 184.215 & 158.52 & 137.21 \\
333.15 & 145.79 & 129.99 & 110.81 \\
338.15 & 117.99 & 105.97 & 91.00 \\
343.15 & 96.51 & 86.49 & 75.78 \\
348.15 & 79.79 & 71.85 & 63.04 \\
353.15 & 67.46 & 60.79 & 53.47 \\
358.15 & 57.27 & 51.85 & 45.63 \\
363.15 & 49.01 & 44.61 & 39.55 \\
\hline
\end{tabular}

"Standard uncertainty is $u(T)=0.1{ }^{\circ} \mathrm{C}$. Expanded uncertainty for the viscosity $U(\eta)=0.02 \mathrm{mPa} . \mathrm{s}(0.95$ level of confidence $)$. 
Table S3. Experimental Data of Electrical Conductivity $\left(\mathrm{mS} . c m^{-1}\right)$ of the Studied DILs*

\begin{tabular}{ccc}
\hline & \multicolumn{2}{c}{ DILs } \\
\cline { 2 - 3 } $\mathrm{T}(\mathrm{K})$ & {$\left[\mathrm{C}_{3}(\mathrm{mim})_{2}\right]\left[\mathrm{NTf}_{2}\right]_{2}$} & {$\left[\mathrm{C}_{5}(\mathrm{mim})_{2}\right]\left[\mathrm{NTf}_{2}\right]_{2}$} \\
\hline 298.15 & 0.44 & 0.60 \\
303.15 & 0.72 & 0.82 \\
308.15 & 0.98 & 1.08 \\
313.15 & 1.31 & 1.41 \\
318.15 & 1.67 & 1.72 \\
323.15 & 2.04 & 2.10 \\
328.15 & 2.56 & 2.57 \\
333.15 & 3.05 & 3.04 \\
338.15 & 3.75 & 4.01 \\
343.15 & 4.28 & 4.91 \\
348.15 & 4.73 & 5.49 \\
353.15 & 5.25 & 5.93 \\
\hline Standard uncertainty is $u(T)=0.1^{\circ} \mathrm{C}$. Expanded uncertainty for the
\end{tabular}

"Standard uncertainty is $u(T)=0.1{ }^{\circ} \mathrm{C}$. Expanded uncertainty for the electrical conductivity is $U(\sigma)=0.02 \mathrm{mS} \cdot \mathrm{cm}^{-1}$ ( 0.95 level of confidence). 
Table S4. Fitted Parameters, Coefficient of Determination, $\mathbf{R}^{2}$, and the Statistical Parameters of the VFT Equation for the Electrical Conductivity of the Studied DILs

\begin{tabular}{|c|c|c|}
\hline & {$\left[\mathrm{C}_{3}(\mathrm{mim})_{2}\right]\left[\mathrm{NTf}_{2}\right]_{2}$} & {$\left[\mathrm{C}_{5}(\mathrm{mim})_{2}\right]\left[\mathrm{NTf}_{2}\right]_{2}$} \\
\hline \multicolumn{3}{|c|}{ VFT equation: $\sigma=A \mathrm{e}^{B /\left(T-T_{0}\right)}$} \\
\hline$A / \mathrm{mS} . \mathrm{cm}^{-1}$ & 263.6755 & 318.0113 \\
\hline$B / \mathrm{K}$ & -585.4288 & -570.6740 \\
\hline$T_{0} / \mathrm{K}$ & 202.3 & 208.5 \\
\hline$R^{2}$ & 0.9953 & 0.9931 \\
\hline bias $\%$ & 0.0407 & -0.0089 \\
\hline$A A D$ & 0.0585 & 0.0459 \\
\hline
\end{tabular}


Table S5. Experimental Data of Density $\left({\left.\mathrm{g} . \mathrm{cm}^{-3}\right) \text { of the Studied DILs }}^{*}\right.$

\begin{tabular}{cccc}
\hline & \multicolumn{3}{c}{ DILs } \\
\cline { 2 - 4 } $\mathrm{T}(\mathrm{K})$ & {$\left[\mathrm{C}_{3}\left(\mathrm{mim}_{2}\right]\left[\mathrm{NTf}_{2}\right]_{2}\right.$} & {$\left[\mathrm{C}_{4}(\operatorname{mim})_{2}\right]\left[\mathrm{NTf}_{2}\right]_{2}$} & {$\left[\mathrm{C}_{5}(\operatorname{mim})_{2}\right]\left[\mathrm{NTf}_{2}\right]_{2}$} \\
\hline 298.15 & 1.6214 & 1.5922 & 1.5704 \\
303.15 & 1.6159 & 1.5867 & 1.5657 \\
308.15 & 1.6114 & 1.5824 & 1.5615 \\
313.15 & 1.6063 & 1.5773 & 1.5575 \\
318.15 & 1.6013 & 1.5724 & 1.5520 \\
323.15 & 1.5965 & 1.5677 & 1.5477 \\
328.15 & 1.5922 & 1.5635 & 1.5441 \\
333.15 & 1.5863 & 1.5589 & 1.5398 \\
\hline
\end{tabular}

Standard uncertainty is $u(T)=0.01{ }^{\circ} \mathrm{C}$. Expanded uncertainty for the density is $U(\rho)=4 \times 10^{-4} \mathrm{~g} \cdot \mathrm{cm}^{-3}(0.95$ level of confidence $)$. 
Table S6. Fitted Parameters, Coefficients of Determination, $\mathbf{R}^{2}$, and the Statistical Parameters of Fitting for the Doolittle Equation (eq 13), Orrik-Erbar Equation (eq 14), and Krossing et al. Equation (eq 15) for the Studied DILs

\begin{tabular}{|c|c|c|c|}
\hline & {$\left[\mathrm{C}_{3}(\mathrm{mim})_{2}\right]\left[\mathrm{NTf}_{2}\right]_{2}$} & {$\left[\mathrm{C}_{4}(\mathrm{mim})_{2}\right]\left[\mathrm{NTf}_{2}\right]_{2}$} & {$\left[\mathrm{C}_{5}(\mathrm{mim})_{2}\right]\left[\mathrm{NTf}_{2}\right]_{2}$} \\
\hline \multicolumn{4}{|c|}{ Doolittle equation: $\eta=a \exp (b /(1-c \rho))$} \\
\hline a. $10^{-7} / \mathrm{Pa} . \mathrm{s}$ & 9.0913 & 7.1091 & 5.9934 \\
\hline$B$ & 4.249 & 4.7240 & 4.7929 \\
\hline$c / \mathrm{cm}^{3} \cdot \mathrm{g}^{-1}$ & 0.76048 & 0.7905 & 0.8072 \\
\hline$R^{2}$ & 0.9936 & 0.9954 & 0.9948 \\
\hline bias & 0.0466 & 0.0295 & 0.0293 \\
\hline$A A D$ & 0.0808 & 0.0567 & 0.0599 \\
\hline \multicolumn{4}{|c|}{ Orrick-Erbar-type equation: $\ln \frac{\eta}{\rho M_{W}}=A+\frac{B}{T}$} \\
\hline$A$ & -19.3106 & -18.1721 & -18.2245 \\
\hline$B / \mathrm{K}$ & 5711.41 & 5291.84 & 5259.88 \\
\hline$R^{2}$ & 0.9974 & 0.9980 & 0.9978 \\
\hline bias & -0.0556 & -0.0082 & -0.0052 \\
\hline$A A D$ & 0.0809 & 0.0249 & 0.0220 \\
\hline \multicolumn{4}{|c|}{ Krossing et al. equation: $\sigma=c e^{-d V_{m}}$} \\
\hline C. $10^{+36} / \mathrm{mS} \mathrm{cm}^{-1}$ & 7.3054 & ----- & 4.7163 \\
\hline$d / \mathrm{mol} \mathrm{cm}^{-3}$ & -0.1699 & ----- & -0.1592 \\
\hline$R^{2}$ & 0.9767 & ----- & 0.9759 \\
\hline Bias & -0.0129 & ----- & 0.0675 \\
\hline$A A D$ & 0.0868 & ---- & 0.0811 \\
\hline
\end{tabular}

